\title{
Super $H$-Antimagic Total Covering for Generalized Antiprism and Toroidal Octagonal Map
}

\author{
Amir Taimur, ${ }^{1}$ Gohar Ali $\left(\mathbb{D},{ }^{1}\right.$ Muhammad Numan, ${ }^{2}$ Adnan Aslam $\left(\mathbb{D},{ }^{3}\right.$ \\ and Kraidi Anoh Yannick (iD) \\ ${ }^{1}$ Department of Mathematics, Islamia College, Peshawar, Pakistan \\ ${ }^{2}$ Department of Mathematics, COMSATS University Islamabad, Attock, Pakistan \\ ${ }^{3}$ Department of Natural Sciences and Humanities, University of Engineering and Technology, Lahore (RCET), Lahore, Pakistan \\ ${ }^{4}$ UFR of Mathematics and Computer Science, University Felix Houphouet Boigny of Cocody, Abidjan, Côte d'Ivoire
}

Correspondence should be addressed to Kraidi Anoh Yannick; kayanoh2000@yahoo.fr

Received 29 June 2021; Accepted 29 July 2021; Published 9 August 2021

Academic Editor: Ali Ahmad

Copyright ( $) 2021$ Amir Taimur et al. This is an open access article distributed under the Creative Commons Attribution License, which permits unrestricted use, distribution, and reproduction in any medium, provided the original work is properly cited.

Let $G$ be a graph and $H \subseteq G$ be subgraph of $G$. The graph $G$ is said to be $(a, d)-H$ antimagic total graph if there exists a bijective function $f: V(H) \cup E(H) \longrightarrow\{1,2,3, \ldots,|V(H)|+|E(H)|\}$ such that, for all subgraphs isomorphic to $H$, the total $H$ weights $W(H)=W(H)=\sum_{x \in V(H)} f(x)+\sum_{y \in E(H)} f(y)$ forms an arithmetic sequence $a, a+d, a+2 d, \ldots, a+(n-1) d$, where $a$ and $d$ are positive integers and $n$ is the number of subgraphs isomorphic to $H$. An $(a, d)$ - $H$ antimagic total labeling $f$ is said to be super if the vertex labels are from the set $\{1,2, \ldots, \mid V(G)\}$. In this paper, we discuss super $(a, d)-C_{3}$-antimagic total labeling for generalized antiprism and a super $(a, d)-C_{8}$-antimagic total labeling for toroidal octagonal map.

\section{Introduction}

All the graphs that we consider in this works are finite, simple, and connected. Let $G$ be a graph with vertex set and edge set denoted by $V(G)$ and $E(G)$, respectively. For the cardinality of vertex set and edge set, we use the notation $|V(G)|$ and $|E(G)|$, respectively. For basic definitions and terminology related to graph theory, the readers can see the book by Gross et al. [1].

A graph labeling is a map $f$ that sends some of the graph elements (vertices or edges or both) to the set of positive integers. If the domain set of $f$ is the set of vertices (edges), then $f$ is called vertex (edge) labeling. If the domain set is $V(G) \cup E(G)$, then $f$ is called total labeling. Let $G$ be a graph and $H_{1}, H_{2}, \ldots, H_{k}$ be subgraphs of $G$. We say that the graph $G$ has an $H_{1}, H_{2}, \ldots, H_{k}$ covering if each edge of $G$ belongs to at least one of the subgraph $H_{i}$, where $1 \leq i \leq k$. If all $H_{i}, i=1,2, \ldots, k$, are isomorphic to a graph $H$, then such a covering is called $H$ covering of $G$. Suppose that a graph $G$ admits an $H$ covering. The graph $G$ is called $(a, d) H$ antimagic if there exists a bijective function $f: V(H) \cup E(H) \longrightarrow\{1,2,3, \ldots,|V(H)|+|E(H)|\} \quad$ such that, for all subgraphs isomorphic to $H$, the total $H$ weights,

$$
W(H)=W(K)=\sum_{x \in V(K)} f(x)+\sum_{y \in E(K)} f(y),
$$

form an arithmetic sequence $a, a+d, a+2 d, \ldots$, $a+(n-1) d$, where $a$ and $d$ are positive integers and $n$ is the number of subgraphs isomorphic to $H$. An $(a, d)-H$ antimagic total labeling $f$ is said to be super if the vertex labels are from the set $\{1,2, \ldots, \mid V(G)\}$. If $d=0$, then $H$ is called $(a, d)-H$ antimagic.

Kotzig and Rosa [2] and Enomoto et al. [3] introduced the concept of edge-magic and super edge-magic labeling. Gutierrez and Llado [4] first studied the $H$ (super) magic coverings of a graph $G$. They proved that the cycle $C_{n}$ and path $P_{n}$ are $P_{m}$ super magic for some $m$. The cycle (super) magic behavior of some classes of connected graphs is studied in Llado et al. [5]. They proved that prisms, 
windmills, wheels, and books are $C_{m}$-magic for some $m$. Maryati et al. [6] investigated the $G$-supermagicness of a disjoint union of $c$ copies of a graph $G$ and showed that the disjoint union of any paths is $c P_{m}$-supermagic for some $c$ and $m$. Maryati et al. [7] and Salman et al. [8] proved that certain families of trees are path-supermagic. Ngurah et al. [9] proved that triangles, chains, ladders, wheels, and grids are cycle-supermagic.

Inaya et al. [10] firstly introduced the concept of $H$-magic decomposition and $H$-antimagic decomposition. They showed that, for any graceful tree $T$ with $n$ edges, the complete graph $K_{2 n+1}$ admits $(a, d)-T$ antimagic decomposition for some $a$ and all even differences $0 \leq d \leq n+1$. They also proved that if any tree $T$ with $n$ edges admits $\alpha$ labeling, then the complete bipartite graph $K_{n, n}$ admits an $(a, d)-T$ antimagic decomposition for some $a$ and $d$ having same parity as $n$. The condition on the existence of $C_{2 k}$ super magic decomposition of complete $n$ partite graph and its copies were given by Lian [11]. The $H$-supermagic decomposition of antiprisms is described by Hendy in [12] and the $H$-supermagic decompositions of the lexicographic product of graphs are discussed by Hendy et al. in [13]. In [14], Hendy et al. examined the existence of super $(a, d)-H$ magic labeling for toroidal grids and toroidal triangulations. Recently, Fenovcikova et al. [15] proved that wheels are cycle antimagic.

In this paper, we discuss the Super $(a, d)$ - $C_{3}$-antimagic total labeling for generalized antiprism and a Super $(a, d)-C_{8}$-antimagic total labeling for toroidal octagonal map. We proved that the generalized antiprism $\mathbb{A}_{r}^{s}$ admits $(a, d)-C_{3}$-antimagic total labeling for $d=0,1$ and the toroidal octagonal map $O_{s}^{r}$ admits a Super $(a, d)-C_{8}$-antimagic total labeling, for $d=1,2, \ldots, 7$.

\section{Results on Super $(a, d)-C_{3}$-Antimagic Total Covering of Generalized Antiprism $\mathbb{A}_{r}^{s}$}

An $r$-sided generalized antiprism $\mathbb{A}_{r}^{s}$ is defined as a polyhedron which is composed of $s$ parallel copies of some particular $r$-sided polygon and connected by an alternating band of triangles. Figure 1 represents the labeled graph of generalized antiprism $A_{r}^{s}$. We denote its vertex set and edge set by $V\left(\mathbb{A}_{r}^{s}\right)$ and $E\left(\mathbb{A}_{r}^{s}\right)$, respectively. The vertex set and the edge set of the generalized antiprism $\mathbb{A}_{r}^{s}$ can be defined as follows:

$$
\begin{aligned}
V\left(\mathbb{A}_{r}^{s}\right)= & \left\{x_{i}^{j}, \text { for } 0 \leq i \leq r-1,0 \leq j \leq s-1\right\} \\
E\left(\mathbb{A}_{r}^{s}\right)= & \left\{x_{i}^{j} x_{i+1}^{j}, \text { for } 0 \leq i \leq r-1,0 \leq j \leq s-1\right\} \\
& \cup\left\{x_{i}^{j} x_{i}^{j+1}, \text { for } 0 \leq i \leq r-1,0 \leq j \leq s-2\right\} \\
& \cup\left\{x_{i}^{j} x_{i+1}^{j+1}, \text { for } 0 \leq i \leq r-1,0 \leq j \leq s-2\right\} .
\end{aligned}
$$

The generalized antiprism $\mathbb{A}_{r}^{s}$ admits a $C_{3}$ covering. Let $z_{i}^{j}$ and $f_{i}^{j}$ be the $C_{3}$ cycles which cover $\mathbb{A}_{r}^{s}$, where $0 \leq i \leq r-1$ and $0 \leq j \leq s-2$. The cycles $z_{i}^{j}$ and $f_{i}^{j}$ can be defined as

$$
\begin{array}{ll}
z_{i}^{j}=x_{i}^{j} x_{i+1}^{j} x_{i+1}^{j+1} x_{i}^{j}, & \text { for } 0 \leq i \leq r-1,0 \leq j \leq s-2, \\
f_{i}^{j}=x_{i}^{j} x_{i+1}^{j+1} x_{i}^{j+1} x_{i}^{j}, & \text { for } 0 \leq i \leq r-1,0 \leq j \leq s-2 .
\end{array}
$$

It is easy to observe that $\left|V\left(\mathbb{A}_{r}^{s}\right)\right|=r s$ and $\left|E\left(\mathbb{A}_{r}^{s}\right)\right|=3 r s-2 r$. We first give an upper bound for $d$ such that $\mathbb{A}_{r}^{s}$ admits a super $(a, d)-C_{3}$-antimagic covering.

Theorem 1. Let $r, s \geq 3$ and $\mathbb{A}_{r}^{s}$ be generalized antiprism graph. Then, there is no super $(a, d)-C_{3}$-antimagic covering with $d \geq 6$.

Proof. Suppose that $\mathbb{A}_{r}^{s}$ has a super $(a, d)-C_{3}$-antimagic covering. Let $f: V\left(\mathbb{A}_{r}^{s}\right) \cup E\left(\mathbb{A}_{r}^{s}\right) \longrightarrow\{1,2,3, \ldots, 4 r s-2 r\}$ be a super $(a, d)-C_{3}$-antimagic covering and $\left\{a_{3}, a_{3}+d, a_{3}+\right.$ $\left.2 d, \ldots, a_{3}+(2 r s-2 r-1) d\right\}$ be the set of $C_{3}$ weights. The minimum weight on cycle $C_{3}$ is at least $12+3 r s$ which is the sum of the smallest vertex labels $(1,2,3)$ and sum of smallest edge labels $(r s+1, r s+2, r s+3)$. Thus,

$$
a_{3} \geq 12+3 r s \text {. }
$$

On the contrary, the maximum possible $C_{3}$-weight is the sum of three largest possible vertex labels, namely, $r s-2, r s-1, r s$, and three the largest possible edge labels from the set, $\{4 r s-2 r-2,4 r s-2 r-1,4 r s-2 r\}$. Hence, we have

$$
a_{3}+(2 r s-2 r-1) d \leq 15 r s-6 r-6 .
$$

From (4) and (5), an upper bound for the parameter $d$ can be obtained as

$$
\begin{aligned}
& d \leq \frac{12 r s-16 r-18}{2 r s-2 r-1}, \\
& d \leq 6-\frac{4 r+6}{2 r s-2 r-1},
\end{aligned}
$$

$$
d \leq 6 .
$$

Thus, we have arrived at the desired result.

Theorem 2. Let $r, s \geq 3$; then, the generalized antiprism $\mathbb{A}_{r}^{s}$ admits a super $(9 r s-3 r+4, o)-C_{3}$-antimagic total covering.

Proof. Let $\phi: V\left(\mathbb{A}_{r}^{s}\right) \cup E\left(\mathbb{A}_{r}^{s}\right) \longrightarrow\{1,2,3, \ldots, 4 r s-2 r\}$ be a total labeling of generalized antiprism $\mathbb{A}_{r}^{s}$ defined as follows:

$$
\begin{aligned}
\phi\left(x_{i}^{j}\right) & =\{j r+1+i, \quad \text { for } 0 \leq i \leq r-1,0 \leq j \leq s-1, \\
\phi\left(x_{i}^{j} x_{i+1}^{j}\right) & =\{(2 s-j) r-i, \quad \text { for } 0 \leq i \leq r-1,0 \leq j \leq s-1, \\
\phi\left(x_{i}^{j} x_{i}^{j+1}\right) & =\{(3 s-2-j) r+r-i, \quad \text { for } 0 \leq i \leq r-1,0 \leq j \leq s-2, \\
\phi\left(x_{i}^{j} x_{i+1}^{j+1}\right) & = \begin{cases}(4 s-3-j) r+r-i, & \text { for } 0 \leq i \leq r-2,0 \leq j \leq s-2, \\
(4 s-3-j) r+1, & \text { for } i=r-1,0 \leq j \leq s-2 .\end{cases}
\end{aligned}
$$

Under the labeling $\phi$, the weights of 3 - cycles $z_{i}^{j}$ are 


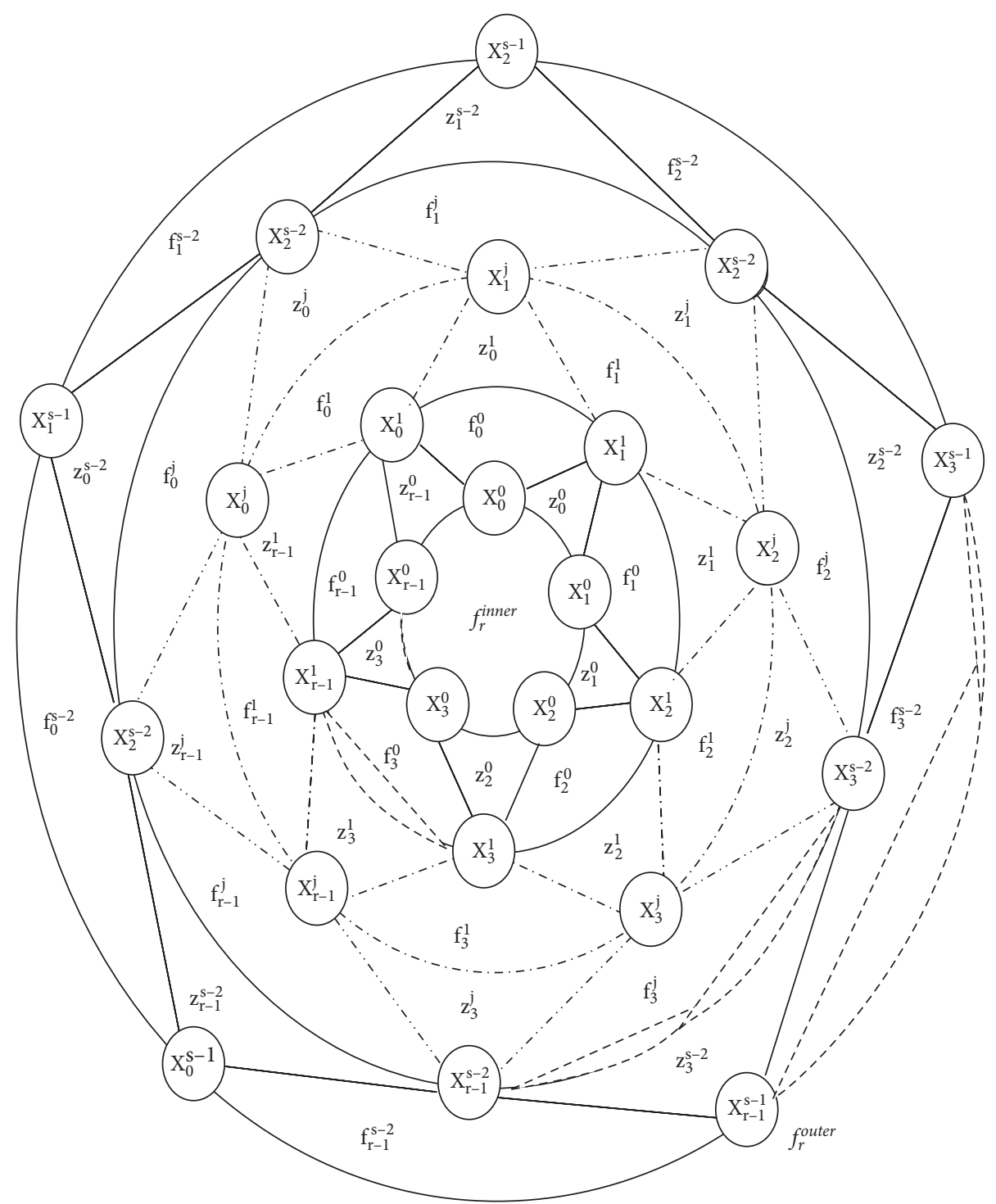

FIgURE 1: Generalized antiprism $\mathbb{A}_{r}^{s}$.

$$
\begin{aligned}
& W\left(z_{i}^{j}\right)=\phi\left(x_{i}^{j}\right)+\phi\left(x_{i+1}^{j}\right)+\phi\left(x_{i+1}^{j+1}\right)+\phi\left(x_{i}^{j} x_{i+1}^{j}\right)+\phi\left(x_{i+1}^{j} x_{i+1}^{j+1}\right)+\phi\left(x_{i}^{j} x_{i+1}^{j+1}\right), \\
& W\left(z_{i}^{j}\right)=\{9 r s-3 r+4, \quad \text { for } 0 \leq i \leq r-1,0 \leq j \leq s-2
\end{aligned}
$$

And, the weights of 3-cycles $f_{i}^{j}$ are

$$
\begin{aligned}
& W\left(f_{i}^{j}\right)=\phi\left(x_{i}^{j}\right)+\phi\left(x_{i+1}^{j+1}\right)+\phi\left(x_{i}^{j+1}\right)+\phi\left(x_{i}^{j} x_{i+1}^{j+1}\right)+\phi\left(x_{i+1}^{j+1} x_{i}^{j+1}\right)+\phi\left(x_{i}^{j+1} x_{i}^{j}\right), \\
& W\left(f_{i}^{j}\right)=\{9 r s-3 r+4, \quad \text { for } 0 \leq i \leq r-1,0 \leq j \leq s-2 .
\end{aligned}
$$


Observe that the weights $W\left(z_{i}^{j}\right)$ and $W\left(f_{i}^{j}\right)$ of all cycles $z_{i}^{j}$ and $f_{i}^{j}$ are equal, and therefore, the resulting labeling is super $(9 r s-3 r+4,0)-C_{3}$ total labeling.

Theorem 3. Let $r, s \geq 3$; then, the generalized antiprism $\mathbb{A}_{r}^{s}$ admits a super $(7 r s+4,2)$-antimagic total covering.

Proof. Let $\chi: V\left(\mathbb{A}_{r}^{s}\right) \cup E\left(\mathbb{A}_{r}^{s}\right) \longrightarrow\{1,2,3, \ldots, 4 r s-2 r\}$ be a total labeling of generalized antiprism $\mathbb{A}_{r}^{s}$ defined as follows.

For $j=$ even, the label on vertices $x_{i}^{j}$ is defined as

$$
\chi\left(x_{i}^{j}\right)= \begin{cases}1+i, & \text { for } 0 \leq i \leq r-1, j=0, \\ (j+1) r, & \text { for } i=0,2 \leq j \leq s-1, \\ j r+i, & \text { for } 1 \leq i \leq r-1,2 \leq j \leq s-1 .\end{cases}
$$

For $j=$ odd, the label on vertices $x_{i}^{j}$ is defined as

$$
\chi\left(x_{i}^{j}\right)= \begin{cases}j r+1, & \text { for } i=0,1 \leq j \leq s-1, \\ (j+1) r+1-i, & \text { for } 1 \leq i \leq r-1,1 \leq j \leq s-1 .\end{cases}
$$

For $j=$ even, the label on edges $\left(x_{i}^{j} x_{i+1}^{j}\right)$ is defined as $\chi\left(x_{i}^{j} x_{i+1}^{j}\right)= \begin{cases}r s+1+i, & \text { for } 0 \leq i \leq r-1, j=0, \\ r s+(j+1) r, & \text { for } i=0,2 \leq j \leq s-1, \\ r s+j r+i, & \text { for } 1 \leq i \leq r-1,2 \leq j \leq s-1 .\end{cases}$

For $j=$ odd, the label on edges $\left(x_{i}^{j} x_{i+1}^{j}\right)$ is defined as $\chi\left(x_{i}^{j} x_{i+1}^{j}\right)= \begin{cases}r s+j r+1, & \text { for } i=0,1 \leq j \leq s-1, \\ r s+(j+1) r+1-i, & \text { for } 1 \leq i \leq r-1,1 \leq j \leq s-1 .\end{cases}$

The label on edges $\left(x_{i}^{j} x_{i}^{j+1}\right)$ is defined as $\chi\left(x_{i}^{j} x_{i}^{j+1}\right)= \begin{cases}(3 s-2) r+1+i, & \text { for } 0 \leq i \leq r-1, j=0, \\ (3 s-1-j) r, & \text { for } i=0,1 \leq j \leq s-1, \\ (3 s-2-j) r+i, & \text { for } 1 \leq i \leq r-1,1 \leq j \leq s-1 .\end{cases}$

And, the label on edges $\left(x_{i}^{j} x_{i+1}^{j+1}\right)$ is defined as $\chi\left(x_{i}^{j} x_{i+1}^{j+1}\right)=3 r s+j r-i, \quad$ for $0 \leq i \leq r-1,0 \leq j \leq s-2$.

Under the labeling $\chi$, the weights of 3-cycle $z_{i}^{j}$ are

$$
\begin{aligned}
W\left(z_{i}^{j}\right)= & \chi\left(x_{i}^{j}\right)+\chi\left(x_{i+1}^{j}\right)+\chi\left(x_{i+1}^{j+1}\right)+\chi\left(x_{i}^{j} x_{i+1}^{j}\right) \\
& +\chi\left(x_{i+1}^{j} x_{i+1}^{j+1}\right)+\chi\left(x_{i}^{j} x_{i+1}^{j+1}\right) .
\end{aligned}
$$

For $j=$ even, we have

$$
W\left(z_{i}^{j}\right)= \begin{cases}7 r s+8+2 i, & \text { for } 0 \leq i \leq r-2, j=0, \\ 7 r s+4, & \text { for } i=r-1, j=0, \\ 7 r s+4 j r+2 r+2, & \text { for } i=0,2 \leq j \leq s-2, \\ 7 r s+4 j r+2+2 i, & \text { for } 1 \leq i \leq r-1,2 \leq j \leq s-2 .\end{cases}
$$

For $j=$ odd, we have

$W\left(z_{i}^{j}\right)= \begin{cases}7 r s+4 j r+4, & \text { for } i=0,1 \leq j \leq s-2, \\ 7 r s+4 j r+2 r+4-2 i, & \text { for } 1 \leq i \leq r-1,1 \leq j \leq s-2 .\end{cases}$

The weight of 3-cycle $f_{i}^{j}$ are

$$
\begin{aligned}
W\left(f_{i}^{j}\right)= & \chi\left(x_{i}^{j}\right)+\chi\left(x_{i+1}^{j+1}\right)+\chi\left(x_{i}^{j+1}\right)+\chi\left(x_{i}^{j} x_{i+1}^{j+1}\right) \\
& +\chi\left(x_{i+1}^{j+1} x_{i}^{j+1}\right)+\chi\left(x_{i}^{j+1} x_{i}^{j}\right) .
\end{aligned}
$$

For $j=$ even, we have

$W\left(f_{i}^{j}\right)= \begin{cases}7 r s+2 r+4, & \text { for } i=0, j=0, \\ 7 r s+4 r+4-2 i, & \text { for } 1 \leq i \leq r-1, j=0, \\ 7 r s+4 j r+4 r+2-2 i, & \text { for } 0 \leq i \leq r-1,2 \leq j \leq s-2 .\end{cases}$

For $j=$ odd, we have

$W\left(f_{i}^{j}\right)= \begin{cases}7 r s+4 j r+4 r+2, & \text { for } i=0,1 \leq j \leq s-2, \\ 7 r s+4 j r+2 r+2+2 i, & \text { for } 1 \leq i \leq r-1,1 \leq j \leq s-2 .\end{cases}$

Observe that the weights $W\left(z_{i}^{j}\right)$ and $W\left(f_{i}^{j}\right)$ form an arithmetic progression with common difference 2 starting from $7 r s+4,7 r s+6$ and ending at $11 r s-4 r+2$. This implies that the defined labeling is a super $(7 r s+4,2)-C_{3}$-antimagic total covering.

\section{Results on Super $(a, d)-C_{8}$-Antimagic Total Covering of Toroidal Octagonal Planner Map $O_{s}^{r}$}

A planar octagonal map is a graph obtained by joining octagons and squares in such a way that they cover the plane. To obtain the toroidal octagonal map, we apply torus identification on octagonal planner map. We denote the toroidal octagonal map with $r$ rows and $s$ column of octagons by $O_{s}^{r}$, where $s, r \geq 2$. The planar representation of $O_{s}^{r}$ is depicted in Figure 2. The vertex set $V\left(O_{s}^{r}\right)$ and the edge set $E\left(O_{s}^{r}\right)$ of octagonal planner map $O_{s}^{r}$ can be defined as follows:

$$
\begin{aligned}
V\left(O_{s}^{r}\right)= & \left\{u_{i}^{j}, v_{i}^{j}, w_{i}^{j}, x_{i}^{j} ; 0 \leq i \leq r-1 \text { and } 0 \leq j \leq s-1\right\}, \\
E\left(O_{s}^{r}\right)= & \left\{u_{i}^{j} v_{i}^{j}, w_{i}^{j} x_{i}^{j} ; 0 \leq i \leq r-1 \text { and } 0 \leq j \leq s-1\right\} \\
& \cup\left\{w_{i}^{j} u_{i}^{j-1} ; 1 \leq i \leq s-1 \text { and } 0 \leq j \leq r-1\right\} \\
& \cup\left\{w_{i}^{0} u_{i}^{s-1} ; 0 \leq i \leq r-1\right\} \\
& \cup\left\{v_{i}^{j} w_{i+1}^{j+1} ; 0 \leq i \leq r-1 \text { and } 0 \leq j \leq s-2\right\} \\
& \cup\left\{v_{i}^{n-1} w_{i+1}^{0} ; 0 \leq i \leq r-1\right\} \\
& \cup\left\{v_{i}^{j} x_{i+1}^{j} ; 0 \leq i \leq r-1 \text { and } 0 \leq j \leq s-1\right\} \\
& \cup\left\{u_{i}^{j} x_{i}^{j} ; 0 \leq i \leq r-1 \text { and } 0 \leq j \leq s-1\right\} .
\end{aligned}
$$




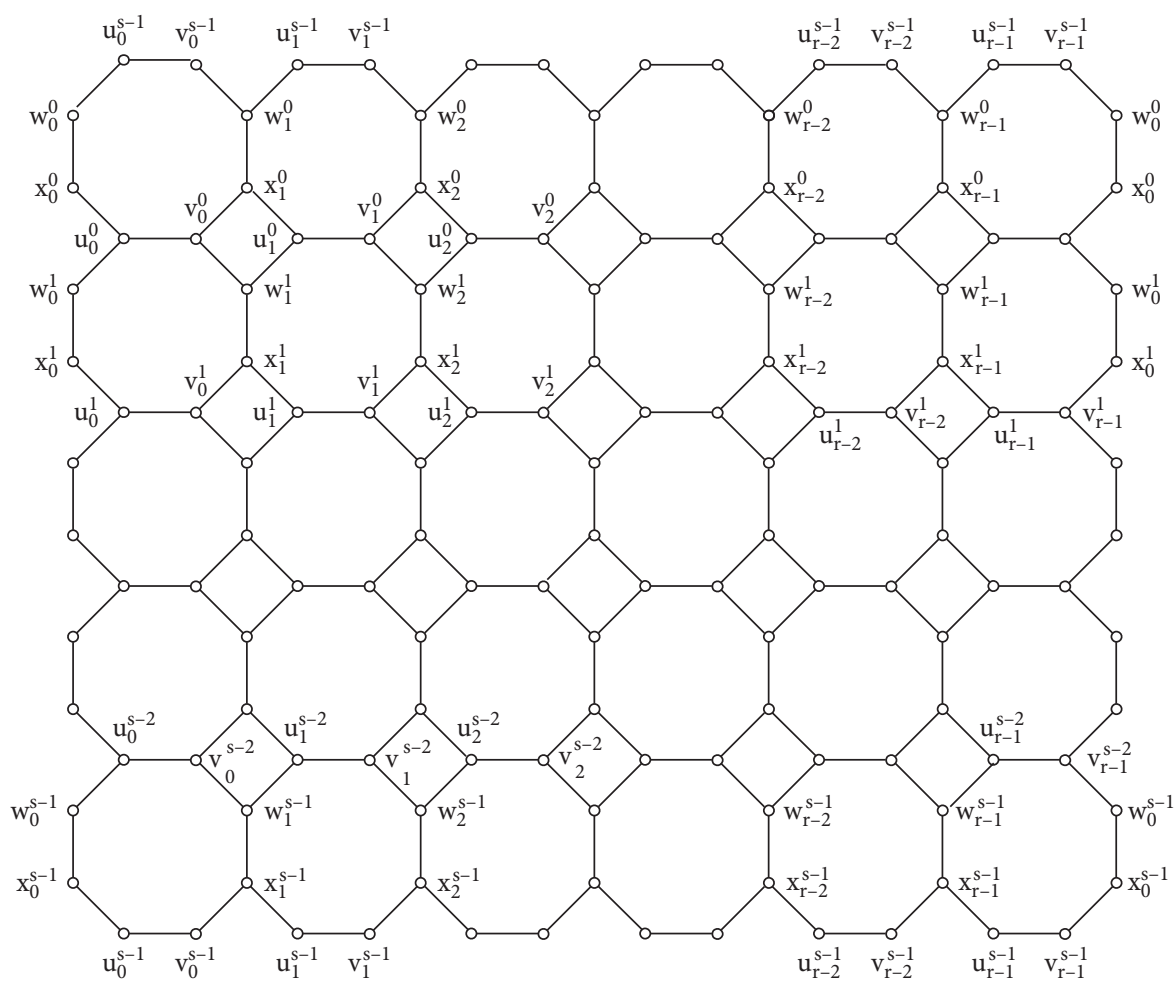

FIgURE 2: Toroidal octagonal map identification $\mathrm{O}_{s}^{r}$

From the above sets, we have $\left|V\left(O_{s}^{r}\right)\right|=4 r s$ and $\left|E\left(O_{s}^{r}\right)\right|=6 r s$. We can cover the toroidal octagonal map $O_{s}^{r}$ by the 8 -sided cycles $C_{8, i}^{j}$. For $0 \leq j \leq s-1$ and $0 \leq i \leq r-1$, the vertex set and edge set of 8 -sided cycles $C_{8, i}^{j}$ can be defined as

$$
\begin{aligned}
& V\left(C_{8, i}^{j}\right)=\left\{w_{i}^{j}, u_{i}^{j-1}, v_{i}^{j-1}, w_{i+1}^{j}, x_{i+1}^{j}, v_{i}^{j}, u_{i}^{j}, x_{i}^{j} ; 0 \leq i \leq r-1,1 \leq j \leq s-1\right\}, \\
& E\left(C_{8, i}^{j}\right)=\left\{w_{i}^{j} u_{i}^{j-1}, u_{i}^{j-1} v_{i}^{j-1}, v_{i}^{j-1} w_{i+1}^{j}, w_{i+1}^{j} x_{i+1}^{j}, v_{i}^{j} x_{i+1}^{j}, u_{i}^{j} v_{i}^{j}, x_{i}^{j} u_{i}^{j}, x_{i}^{j} w_{i}^{j} ; 0 \leq i \leq r-1,1 \leq j \leq s-1\right\}, \\
& V\left(C_{8, i}^{0}\right)=\left\{w_{i}^{0}, u_{i}^{s-1}, v_{i}^{s-1}, w_{i+1}^{0}, x_{i+1}^{0}, v_{i}^{0}, u_{i}^{0}, x_{i}^{0} ; 0 \leq i \leq r-1,\right\}, \\
& E\left(C_{8, i}^{0}\right)=\left\{w_{i}^{0} u_{i}^{s-1}, u_{i}^{s-1} v_{i}^{s-1}, v_{i}^{s-1} w_{i+1}^{0}, w_{i+1}^{0} x_{i+1}^{0}, v_{i}^{0} x_{i+1}^{0}, u_{i}^{0} v_{i}^{0}, x_{i}^{0} u_{i}^{0}, x_{i}^{0} w_{i}^{0} ; 0 \leq i \leq s-1\right\} .
\end{aligned}
$$

We start by giving an upper bound for $d$ such that $O_{s}^{r}$ admits a super $(a, d)-C_{8}$-antimagic covering.

Theorem 4. Suppose $O_{s}^{r}$ admits a super $(a, d)-C_{8}$-antimagic covering; then, $d \leq 80$.

Proof. Suppose $O_{s}^{r}$ admits a super $(a, d)-C_{8}$-antimagic covering. Then, the weight on cycle $C_{8}$ is atleast

$$
\sum_{i=1}^{8} i+\sum_{i=1}^{8}(4 r s+i)=32 r s+72,
$$

and the largest weight of $C_{8}$ is atmost

$$
\sum_{i=1}^{8}(4 r s+1-i)+\sum_{i=1}^{8}(10 r s+1-i)=112 r s-56 .
$$

Thus, we have

$$
\begin{aligned}
a+(r s-1) d & \leq 112 r s-56, \\
(r s-1) d & \leq 112 r s-56-32 r s-72, \\
d & \leq \frac{80 r s-128}{r s-1},
\end{aligned}
$$

$d \leq 80$.

In the next two theorems, we show that toroidal octagonal map $O_{s}^{r}$ admits a super $(a, d)-C_{8}$-antimagic covering for $d=1,2, \ldots 7$.

Theorem 5. Let $r, s \geq 2$; then, the toroidal octagonal map $O_{s}^{r}$ is super $(a, d)-C_{8}$-antimagic for $d \in\{1,3,5,7\}$. 
Proof. Define a total labeling $\varphi_{d}: V\left(O_{s}^{r}\right) \cup E\left(O_{s}^{r}\right) \longrightarrow$ $\left\{1,2,3, \ldots,\left|V\left(O_{s}^{r}\right)\right|+\left|E\left(O_{s}^{r}\right)\right|\right\}$, where $d \in\{1,3,5,7\}$ as follows:

$$
\begin{aligned}
\varphi_{d}\left(u_{i}^{j}\right) & =j r+1+i, \quad 0 \leq i \leq r-1,0 \leq j \leq s-1, \\
\varphi_{d}\left(v_{i}^{j}\right) & =r s+j r+1+i, \quad 0 \leq i \leq r-1,0 \leq j \leq s-1, \\
\varphi_{d}\left(x_{i}^{j}\right) & =3 r s+(s-1-j) r+r-i, \quad 0 \leq i \leq r-1,0 \leq j \leq s-1, \\
\varphi_{d}\left(w_{i}^{j}\right) & =2 r s+j r+1+i, \quad 0 \leq i \leq r-1,0 \leq j \leq s-1, \\
\varphi_{d}\left(u_{i}^{j} v_{i}^{j}\right) & =4 r s+(s-1-j) r+r-i, \quad 0 \leq i \leq r-1,0 \leq j \leq s-1, \\
\varphi\left(x_{i}^{j} w_{i}^{j}\right) & =5 m n++2 j m+1+2 i, \quad 0 \leq i \leq r-1,0 \leq j \leq s-1, \\
\varphi_{1}\left(v_{i}^{j} x_{i+1}^{j}\right) & =\varphi_{3}\left(v_{i}^{j} x_{i+1}^{j}\right)=8 r s+(s-1-j) r+r-i, \quad 0 \leq i \leq r-1,0 \leq j \leq s-1, \\
\varphi_{5}\left(v_{i}^{j} x_{i+1}^{j}\right) & =\varphi_{7}\left(v_{i}^{j} x_{i+1}^{j}\right)=8 r s+j r+1+i, \quad 0 \leq i \leq r-1,0 \leq j \leq s-1, \\
\varphi_{d}\left(u_{i}^{j} w_{i}^{j+1}\right) & =5 r s+2(s-1-j) r+2 r-2 i, \quad 0 \leq i \leq r-1,0 \leq j \leq s-1, \\
\varphi_{1}\left(v_{i}^{j} w_{i}^{j+1}\right) & =7 r s+(s-1-j) r+r-i, \quad 0 \leq i \leq r-1,0 \leq j \leq s-1, \\
\varphi_{3}\left(v_{i}^{j} w_{i}^{j+1}\right) & =\varphi_{5}\left(v_{i}^{j} w_{i}^{j+1}\right)=\varphi_{7}\left(v_{i}^{j} w_{i}^{j+1}\right)=7 r s+r j+1+i, \quad 0 \leq i \leq r-1,0 \leq j \leq s-1, \\
\varphi_{1}\left(x_{i}^{j} u_{i}^{j}\right) & =\varphi_{3}\left(x_{i}^{j} u_{i}^{j}\right)=\varphi_{5}\left(x_{i}^{j} u_{i}^{j}\right)=9 r s+(s-1-j) r+r-i, \quad 0 \leq i \leq r-1,0 \leq j \leq s-1, \\
\varphi_{7}\left(x_{i}^{j} u_{i}^{j}\right) & =9 r s+j r+1+i, \quad 0 \leq i \leq r-1,0 \leq j \leq s-1 .
\end{aligned}
$$

The total labeling $\varphi_{d}$ labels the vertices $u_{i}^{j}, v_{i}^{j}, w_{i}^{j}, x_{i}^{j}$ from the set $\{1,2, \ldots, 4 r s\}$ and the edges from the set
$\{4 r s+1,4 r s+2, \ldots, 10 r s\}$. For $0 \geq i \geq r-1$ and $0 \geq j \geq s-1$, the weight of cycles $C_{8, i}^{j}$ under $\varphi_{d}$ is

$$
\begin{aligned}
W_{d}\left(C_{8, i}^{j}\right)= & \varphi_{d}\left(u_{i}^{j-1}\right)+\varphi_{d}\left(v_{i}^{j-1}\right)+\varphi_{d}\left(u_{i}^{j-1} v_{i}^{j-1}\right)+\varphi_{d}\left(w_{i+1}^{j}\right)+\varphi_{d}\left(w_{i}^{j}\right)+\varphi_{d}\left(w_{i+1}^{j} v_{i}^{j-1}\right) \\
& +\varphi_{d}\left(x_{i+1}^{j}\right)+\varphi_{d}\left(x_{i+1}^{j} w_{i+1}^{j}\right)+\varphi_{d}\left(v_{i}^{j}\right)+\varphi_{d}\left(v_{i}^{j} x_{i+1}^{j}\right)+\varphi_{d}\left(u_{i}^{j}\right) \\
& +\varphi_{d}\left(u_{i}^{j} v_{i}^{j}\right)+\varphi_{d}\left(x_{i}^{j}\right)+\varphi_{d}\left(x_{i}^{j} u_{i}^{j}\right)+\varphi_{d}\left(x_{i}^{j} w_{i}^{j}\right)+\varphi_{d}\left(w_{i}^{j} u_{i}^{j-1}\right), \\
W_{d}\left(C_{8, i}^{j}\right)= & \begin{cases}68 r s+2 r+10+j r+i, & \text { for } d=1, \\
67 r s+r+11+3 j r+3 i, & \text { for } d=3, \\
66 r s+r+12+5 j r+5 i, & \text { for } d=5, \\
65 r s+13+7 j r+7 i, & \text { for } d=7 .\end{cases}
\end{aligned}
$$

For the case $d=1$, we have weights' set $\{68 r s+2 r+10,68 r s+2 r+11, \ldots, 69 r s+2 r+9\} ;$ similarly, for cases $d=3,5,7$, we get the weights from the sets $\{67 r s+r+11,67 r s+2 r+12, \ldots, 70 r s+r+8\}, \quad\{66 r s+r+$
$12,66 r s+r+17, \ldots, 71 r s+r+7\}, \quad$ and $\quad\{65 r s+r+$ $13,65 r s+r+20, \ldots, 72 r s+r+5\}$, respectively. Hence, the weights of cycles $C_{8, i}^{j}$ form an arithmetic sequence with difference $1,3,5$, and 7 . 
Theorem 6. Let $r, s \geq 2$; then, the toroidal map $O_{s}^{r}$ is super (a,d)-C $C_{8}$-antimagic for $d \in\{2,4,6\}$.
Proof. Let $d \in\{2,4,6\}$ and $0 \leq i \leq r-1,0 \leq j \leq s-1$. We define a total labeling $\phi_{d}$ of $O_{s}^{r}$ as follows:

$$
\begin{aligned}
\phi_{d}\left(u_{i}^{j}\right) & =j r+1+i, \quad 0 \leq i \leq r-1,0 \leq j \leq s-1, \\
\phi_{d}\left(v_{i}^{j}\right) & =r s+j r+1+i, \quad 0 \leq i \leq r-1,0 \leq j \leq s-1, \\
\phi_{d}\left(x_{i}^{j}\right) & =3 r s+(s-1-j) r+r-i, \quad 0 \leq i \leq r-1,0 \leq j \leq s-1, \\
\phi_{d}\left(w_{i}^{j}\right) & =2 r s+j r+1+i, \quad 0 \leq i \leq r-1,0 \leq j \leq s-1, \\
\phi_{d}\left(u_{i}^{j} v_{i}^{j}\right) & =8 r s+(s-1-j) r+r-i, \quad 0 \leq i \leq r-1,0 \leq j \leq s-1, \\
\phi_{2}\left(x_{i}^{j} w_{i}^{j}\right) & =\varphi_{4}\left(x_{i}^{j} w_{i}^{j}\right)=9 r s+(s-1-j) r+r-i, \quad 0 \leq i \leq r-1,0 \leq j \leq s-1, \\
\phi_{6}\left(x_{i}^{j} w_{i}^{j}\right) & =9 r s+j r+1+i, \quad 0 \leq i \leq r-1,0 \leq j \leq s-1, \\
\phi_{2}\left(v_{i}^{j} x_{i+1}^{j}\right) & =\varphi_{4}\left(v_{i}^{j} x_{i+1}^{j}\right)=6 r s+j r+1+i, \quad 0 \leq i \leq r-1,0 \leq j \leq s-1, \\
\phi_{6}\left(v_{i}^{j} x_{i+1}^{j}\right) & =6 r s+(s-1-j) r+r-i, \quad 0 \leq i \leq r-1,0 \leq j \leq s-1, \\
\phi_{d}\left(u_{i}^{j} w_{i}^{j+1}\right) & =4 r s+j r+1+i, \quad 0 \leq i \leq r-1,0 \leq j \leq s-1, \\
\phi_{d}\left(v_{i}^{j} w_{i}^{j+1}\right) & =5 r s+r j+1+i, \quad 0 \leq i \leq r-1,0 \leq j \leq s-1, \\
\phi_{2}\left(x_{i}^{j} u_{i}^{j}\right) & =7 r s+(s-1-j) r+r-i, \quad 0 \leq i \leq r-1,0 \leq j \leq s-1, \\
\phi_{4}\left(x_{i}^{j} u_{i}^{j}\right) & =\phi_{6}\left(x_{i}^{j} u_{i}^{j}\right)=7 r s+j r+1+i, \quad 0 \leq i \leq r-1,0 \leq j \leq s-1 .
\end{aligned}
$$

The total labeling $\phi_{d}$ labels the vertices $u_{i}^{j}, v_{i}^{j}, w_{i}^{j}, x_{i}^{j}$ from the set $\{1,2, \ldots, 4 r s\}$ and edges from the set $\{4 r s+1,4 r s+2, \ldots, 10 r s\}$. This show that $\varphi_{d}$ is a bijection from set $V\left(O_{s}^{r}\right) \cup E\left(O_{s}^{r}\right)$ to set $\{1,2, \ldots, 10 r s\}$. For $1 \geq i \geq l$ and $i \geq j \geq k$, the weights of $C_{8, i}^{j}$ under the labeling $\phi_{d}$ are

$$
\begin{aligned}
W_{d}\left(C_{8, i}^{j}\right)= & \phi_{d}\left(u_{i}^{j-1}\right)+\phi_{d}\left(v_{i}^{j-1}\right)+\phi_{d}\left(u_{i}^{j-1} v_{i}^{j-1}\right)+\phi_{d}\left(w_{i+1}^{j}\right)+\phi_{d}\left(w_{i}^{j}\right)+\phi_{d}\left(w_{i+1}^{j} v_{i}^{j-1}\right) \\
& +\phi_{d}\left(x_{i+1}^{j}\right)+\phi_{d}\left(x_{i+1}^{j} w_{i+1}^{j}\right)+\phi_{d}\left(v_{i}^{j}\right)+\phi_{d}\left(v_{i}^{j} x_{i+1}^{j}\right)+\phi_{d}\left(u_{i}^{j}\right) \\
& +\phi_{d}\left(u_{i}^{j} v_{i}^{j}\right)+\phi_{d}\left(x_{i}^{j}\right)+\phi_{d}\left(x_{i}^{j} u_{i}^{j}\right)+\phi_{d}\left(x_{i}^{j} w_{i}^{j}\right)+\phi_{d}\left(w_{i}^{j} u_{i}^{j-1}\right), \\
W_{d}\left(C_{8, i}^{j}\right)= & \begin{cases}75 r s-4 r+8+2 j r+2 i, & \text { for } d=2, \\
74 r s-4 r+9+4 j r+4 i, & \text { for } d=4, \\
73 r s-4 r+12+6 j r+6 i, & \text { for } d=6 .\end{cases}
\end{aligned}
$$

For the case $d=2$, we have weights from the set $\{75 r s-4 r+8,75 r s-4 r+10, \ldots, 77 r s-4 r+6\}$. Similarly, for cases $d=4,6$, we get weights from the sets $\{74 r s-4 r+9,74 r s-4 r+13, \ldots, 78 r s-4 r+5\}$ and $\{73 r s-$ $4 r+12,73 r s-4 r+18, \ldots, 79 r s-4 r+6\}$, respectively. This showed that weights of the cycles $C_{8, i}^{j}$ form an arithmetic sequence with difference 2,4 , and 6 .

\section{Conclusion}

In the present paper first, we constructed an upper bound for the parameter $d$ for super $(a, d)-C_{3}$-antimagic covering. Secondly, we examined the existence of super $(a, d)-C_{3}$-antimagic labeling of generalized antiprism $\mathbb{A}_{r}^{s}$. We showed that, for $r, s \geq 3$ the generalized antiprism $A_{r}^{s}$ had 
$(a, d)$ - $C_{3}$-antimagic covering for $d \in\{0,2\}$. Thirdly, we constructed an upper bound for the parameter $d$ for super $(a, d)-C_{8}$-antimagic covering. Finally, we examined the existence of super $(a, d)-C_{8}$-antimagic labeling of torodial map $O_{s}^{r}$. We showed that, for $m, n \geq 2$, the torodial octagonal map $O_{s}^{r}$ had $(a, d)$ - $C_{8}$-antimagic covering for $d \in\{1,2,3,4,5,6,7\}$. We conclude the paper with the following open problems.

Open problem 1: find other possible bound for parameter $d$ under $(a, d)-C_{3}$-antimagic total covering and the corresponding remaining labeling of $d$ for generalized antiprism $\mathbb{A}_{r}^{s}$

Open problem 2: find other possible bound for parameter $d$ under $(a, d)-C_{8}$-antimagic total covering and the corresponding remaining labeling of $d$ for torodial octagonal map $O_{s}^{r}$

\section{Data Availability}

No data were used to support the study.

\section{Conflicts of Interest}

The authors declare that they have no conflicts of interest.

\section{References}

[1] J. L. Gross, J. Yellen, and P. Zhang, Handbook of Graph Theory, CRC Press, Taylor and Francis Group, Boca Raton, FL, USA, Second edition, 2014.

[2] A. Kotzig and A. Rosa, "Magic valuations of finite graphs," Canadian Mathematical Bulletin, vol. 13, no. 4, pp. 451-461, 1970.

[3] H. Enomoto, A. S. Llado, T. Nakamigawa, and G. Ringel, "Super edge-magic graphs," SUT Journal of Mathematics, vol. 34, pp. 105-109, 1998.

[4] A. Gutierrez and A. Llado, "Magic coverings," Journal of Combinatorial Mathematics and Combinatorial Computing, vol. 55, pp. 43-46, 2005.

[5] A. Llado and J. Moragas, "Cycle-magic graphs," Discrete Mathematics, vol. 307, no. 29-25, pp. 29-33, 2007.

[6] T. K. Maryati, A. N. M. Salman, and E. T. Baskoro, "Supermagic coverings of the disjoint union of graphs and amalgamations," Discrete Mathematics, vol. 313, no. 4, pp. 397-405, 2013.

[7] T. K. Maryati, A. N. M. Salman, E. T. Baskoro, J. Ryan, and M. Miller, "On $H$-supermagic labelings for certain shackles and amalgamations of a connected graph," Utilitas Mathematica, vol. 83, pp. 333-342, 2010.

[8] A. N. M. Salman, A. A. G. Ngurah, and N. Izzati, "On (super)edge-magic total labelings of subdivision of stars Sn," Utilitas Mathematica, vol. 81, pp. 275-284, 2010.

[9] A. A. G. Ngurah, A. N. M. Salman, and L. Susilowati, "Hsupermagic labelings of graphs," Discrete Mathematics, vol. 310, no. 8, pp. 1293-1300, 2010.

[10] N. Inayah, A. N. M. Salman, and R. Simanjuntak, "On $(a, d)$-H-antimagic coverings of graphs," Journal of Combinatorial Mathematics and Combinatorial Computing, vol. 71, pp. 273-281, 2009.

[11] Z. Liang, "Cycle-supermagic decompositions of complete multipartite graphs," Discrete Mathematics, vol. 312, no. 22, pp. 3342-3348, 2012.
[12] K. A. Hendy, "The $H$-super(anti)magic decomposition of antiprism graphs," AIP Conference Proceedings, vol. 1707, Article ID 020007, 2016.

[13] K. A. Hendy, A. N. Sugeng, and M. Salman, "An H-super magic decompositions of the lexicographic product of graphs," AIP Conference Proceedings, vol. 2023, Article ID 020193, 2018.

[14] K. A. Hendy, A. N. Mudholifah, K. A. Sugeng, M. Baca, and A. Semanicova-Fenovcikova, "On H-antimagic decomposition of toroidal grids and triangulations," AKCE International Journal of Graphs and Combinatorics, vol. 17, no. 3, 2019.

[15] A. S. Fenovcikova, M. Baca, M. Lascsakova, M. Miller, and J. Ryan, "Wheels are cycle-antimagic," Electronic Notes in Discrete Mathematics, vol. 48, pp. 11-18, 2015. 